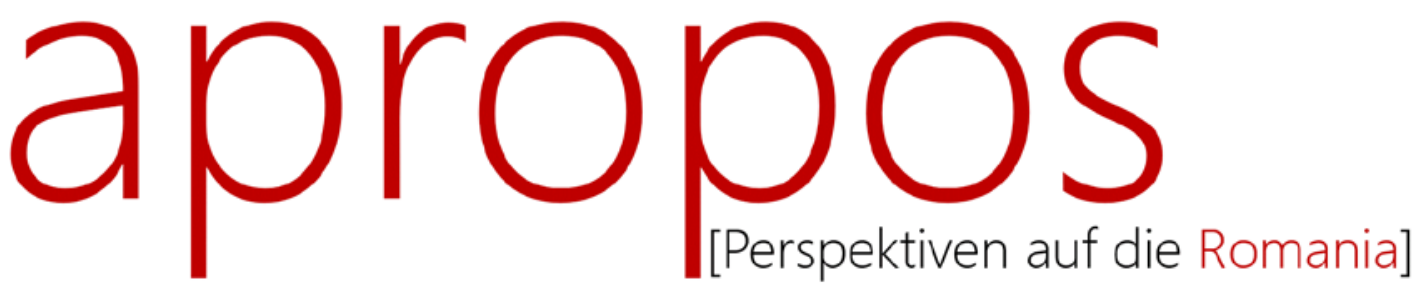

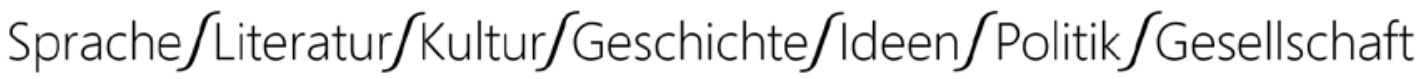

$2 \mid 2019$

Rugbykultur (in) der Romania

Rezension von Wieser, Doris \& Ana Filipa Prata (ed.). 2018. Cities of the Lusophone World. Literature, Culture and Urban Transformations. Oxford et al.: Peter Lang. Alexander Altevoigt

apropos [Perspektiven auf die Romania] hosted by Hamburg University Press

2019, 2

pp. $168-171$

ISSN: $2627-3446$

Online

https://journals.sub.uni-hamburg.de/apropos/article/view/1386

Zitierweise

Altevoigt, Alexander. 2019. „Rezension von Wieser, Doris \& Ana Filipa Prata (ed.). 2018. Cities of the Lusophone World. Literature, Culture and Urban Transformations. Oxford et al.: Peter Lang", apropos [Perspektiven auf die Romania] 2, 168-171. doi: 10.15460/apropos.0.1386

Except where otherwise noted, this article is licensed under a Creative Commons Attribution 4.0 International license (CC BY 4.0)

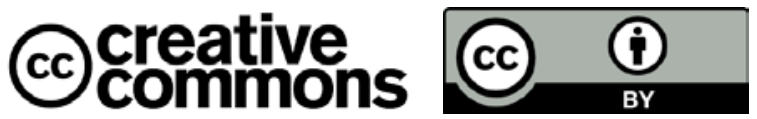




\title{
Alexander Altevoigt
}

Rezension

\section{Wieser, Doris \& Ana Filipa Prata (ed.). 2018. Cities of the Lusophone World. Literature, Culture and Urban Transformations. Oxford et al.: Peter Lang.}

\author{
Alexander Altevoigt \\ ist wissenschaftlicher Mitarbeiter im \\ Bereich der iberoromanischen \\ Literaturwissenschaft am Seminar für \\ Romanische Philologie der Universität \\ Göttingen. \\ alexander.altevoigt@uni-goettingen.de
}

Keywords

Urban Studies - Lusophonie - Stadt - Raumforschung - Postkolonialismus

Mit Cities of the Lusophone World. Literature, Culture and Urban Transformations haben die beiden Literatur- und Kulturwissenschaftlerinnen Doris Wieser und Ana Filipa Prata einen umfangreichen, interdisziplinären Sammelband vorgelegt, der sich dem an der Schnittstelle von Soziologie und Kulturwissenschaften befindlichen Feld der urban cultural studies widmet. In ihrer Einleitung geben sie einen Überblick über rezente Entwicklungen in dieser Disziplin und verorten die Entstehung des Forschungsfelds im größeren Wissenschaftskontext des spatial turn, der solch ausdifferenzierte Studien auch im Jahr 2019 noch nach sich zieht. Dies äußert sich bei den Einzelbeiträgen in den gewählten theoretischen Grundlagen, wenn beispielsweise Nicht-Orte (Marc Augé), Heterotopien (Michel Foucault), Räume von imagined communities (Benedict Anderson) oder koloniale Raumspezifika (Frantz Fanon) zur Sprache kommen. Auch geographisch gesehen bieten die Aufsätze ein breites Panorama: Städtische Räume in Portugal (Lissabon), Brasilien (Porto Alegre, São Paulo), Mosambik (llha de Moçambique, Maputo), Macau, Angola (Luanda) und, weniger stark, Kap Verde werden unter die Lupe genommen. Fünf der Artikel - und damit die Mehrheit - widmen sich urbanen Dynamiken in Lissabon. Möglicher Kritik an einer Überprivilegierung der portugiesischen Hauptstadt bei der Auswahl der Beiträge kommen die beiden Herausgeberinnen indes zuvor: Das Buch könne nur ein Anfang vieler Arbeiten zum Thema sein und biete deshalb lediglich beispielhafte Perspektiven auf ausgewählte urbane Räume der Lusophonie, verankert in verschiedenen Disziplinen. 
Die 13 Artikel des 284 Seiten starken Bandes stammen je zur Hälfte aus der von Wieser und Prata geleiteten Sektion beim Deutschen Lusitanistentag 2015 und aus einem von Doris Wieser initiierten Projekts am Lissaboner Zentrum für Komparatistik (CEC). Auf diese Weise wurden die Fallstudien zusammengetragen, deren Ziel es ist, von konkreten Beispielen der kulturellen Repräsentation oder Lesbarkeit urbaner Prozesse zu abstrahieren, ohne dabei den Anspruch zu erheben, universelle, allgemeingültige Aussagen zu treffen. Dies spiegelt sich auch in der Gliederung des Bandes wider, der nicht etwa nach geographischen Regionen oder kulturellen Ausdrucksformen organisiert ist, sondern in drei thematischen Sektionen: "Framing: Memories and Identities", "Struggling: Margins and Resistance" und "Crossing: Migrations and Transformations". Erfahrungsgemäß ist es sehr schwierig, trennscharfe Unterteilungen bei solchen Publikationen zu realisieren, weshalb es hier und dort zu thematischen Überschneidungen über die gesetzten Grenzen hinaus kommt. So sind soziale Ausgrenzungsprozesse in allen drei Sektionen von Bedeutung. Dies in Kauf zu nehmen ist jedoch allemal besser, als das Risiko einzugehen, mit Blöcken wie „Urbane Dynamiken in Lissabon“ oder „Fotografische Darstellungen der Stadt“ den Eindruck zu vermitteln, der jeweilige Raum oder die Kunstform sei vollständig erfasst.

Den ersten Abschnitt eröffnet Isabel Araújo Branco mit einem Beitrag über die Lissabon-Aufnahmen des Fotografen Eduardo Gageiro. Seine Bilder aus der Zeit der Diktatur erfüllen nicht nur eine Dokumentarfunktion, sondern lassen sich auch als - wenn man so will - photographie engagée neorealistischer Prägung verstehen. Ohne den Ausschnitt- und Konstruktcharakter von Fotografien auszublenden, spricht Branco dem Fotografen das Talent zu, so etwas wie das „wahre Lissabon“ einzufangen. Der zweite Beitrag von Ana Filipa Prata widmet sich der Frage nach der Repräsentation afrikanischer und vor allem schwarzer Bewohner Lissabons. Der von ihr untersuchte Text lotet die Grenzen zwischen Ethnografie und Fiktion aus und präsentiert verschiedene narrative Strategien (Polyphonie, PseudoFeldforschung), um den weißen Schleier, der die Stadt bedeckt, anzuheben. Darunter befinden sich - folgt man Pratas Analyse - widersprüchliche Diskurse und letztendlich die schwierige und weiter auszuhandelnde Frage danach, wem die Stadt gehört. Im Anschluss an Prata verschiebt sich der Fokus weg von Portugal hin nach Brasilien. Anne Burgert interessiert sich für den Schriftsteller Moacyr Scliar und die Darstellung des jüdischen Einwandererstadtteils Bom Fim in zweien seiner Romane aus den 1970ern. Burgert zeigt vor allem zwei Dinge: Dass sich das Postulat der Anonymität in der Großstadt nicht bewahrheiten muss und dass ein Autor geradezu als Formgeber eines urbanen Raums avancieren kann. Die Wahrnehmung des Viertels Bom Fim wurde nämlich maßgeblich von Scliars Texten beeinflusst. Auch dem lusophonen Afrika wird im ersten Abschnitt Beachtung geschenkt, wenn Ana Mafalda Leite und Jessica Falconi aus zweifacher Perspektive (Lyrik und Film) auf die ehemalige mosambikanische (Kolonial-)Hauptstadt Ilha de Moçambique schauen. Leite beschreibt, wie in Gedichten von Rui Knopfli Räume Stimmen bekommen und so zu (post-)kolonialen Figuren werden können. Falconi zieht aus einem Dokumentarfilm über die Insel die Erkenntnis, dass die dem Indischen Ozean zugewandte Stadt durch Musealisierung, Tourismus, Vergangenheitsbewältigung und Identitätskonstruktionen geformt wurde und wird. Mônica Simas schließt den ersten Teil des Bandes mit einem Augenzeugenbericht vom Literaturfestival The 
Script Road, das seit 2012 in Macau stattfindet. Die Autorin hat im Jahr 2015 das Event besucht und diskutiert nun, ob die Veranstaltung die Stadt als open city ausweist. Die monolinguale Situation und die Abwesenheit echten Austausches lassen sie diese Frage verneinen.

Mit dem Aufsatz von Luís Pimenta Lopes über den Debutroman von Pedro Vieira von 2011 beginnt der zweite Teil des Bandes. Er rückt die Frage nach Zentrum und Peripherie in den Fokus. Der Zug, der die Vorstadt mit der Innenstadt verbindet, wird zur Metapher für die Narration selbst und invertiert das Zentrum-PeripherieVerhältnis: Das Zentrum mit seinen Nicht-Orten dient nur der Arbeit, während die Vororte Hauptschauplatz des Lebens sind. Wie kulturell-literarischer Aktivismus (literatura marginal) die sozio-ökonomische Marginalisierung bestimmter urbaner Gruppen kritisch thematisiert und somit der Peripherie wieder Sichtbarkeit und Bedeutung verleiht, analysiert Ingrid Hapke. Sie unterstreicht die konkrete Wechselwirkung zwischen dem urbanen Raum und der Literatur, die in ihm veröffentlicht, performt und rezipiert wird (litera-rua). Júlia Garraio widmet sich Luanda. Mit dem Zuzug vieler Kinder und Jugendlicher nach der Unabhängigkeit wurde die Stadt zu einer Projektionsfläche und zu einem Aushandlungsort der jungen Nation. Garraio analysiert Texte, die die Misere der Kinder zeigen und versuchen, für diese zu sprechen. Als Abschluss der zweiten Sektion liest Christian Grünnagel den Roman Barroco Tropical (2009) von Agualusa als sozialkritischen Text, dessen Konstruktion eines Termitenhügel-Hochhauses und anderer dystopischer Orte sinnbildlich für die Probleme der Metropole in frischer postkolonialer Zeit stehen.

Eröffnet wird der dritte und letzte Abschnitt von Paulo de Medeiros und einer geschichtswissenschaftlichen Analyse von Fotografien, die ein fragmentiertes postimperiales Lissabon zeigen. Medeiros wagt auch den Vergleich mit der Zeit unmittelbar nach 1755, als Lissabon nach einem Erdbeben neu geformt wurde. Cristina Martínez-Tejero stellt verschiedene Formen öffentlicher Kunst vor, die sich aktiv gegen die Vereinnahmung Lissabons durch Tourismus und Neoliberalismus positioniert. Die Stadt wird so selber zu einem semiotic space, an dessen Wände und Mauern drängende Diskurse sichtbar werden. Im Anschluss stellt Doris Wieser den Roman Crónica da Rua 513.2 von João Paulo Borges Coelho vor und analysiert, wie ein einzelner urbaner Straßenzug in Maputo zum Mikrokosmos einer postkolonialen moçambicanidade wird. Besondere Bedeutung erfährt der Spuk des Kolonialismus, der in geisterhaften Figuren aus vergangenen Zeiten Ausdruck findet. Den dritten Teil und damit das ganze Buch beschließt Janek Scholz mit der Interpretation mehrerer Comics aus Portugal, Brasilien, Angola und Kap Verde. Ausgehend vom Konzept der Beschleunigung (Hartmut Rosa) legt Scholz verschiedene Strategien offen, wie die Figuren der Comics auf die fragmentierte postmoderne Stadt reagieren, und zeigt, wie kulturelle Produktionen als Spiegelbild realer demographischer urbaner Entwicklungen verstanden werden können.

Wieser und Prata haben es also geschafft, multidimensionale Perspektiven auf städtische Räume in der Lusophonie zu eröffnen, die es so zusammen in einem Sammelband bisher nicht gab. So werden speziell urbane Dynamiken von Kultur und Literatur ins Bewusstsein gerückt, etwa wie anonyme und kollektive 
Autorschaft durch Kunst im öffentlichen Raum sowie die Gefahren neoliberaler Vereinnahmung von eigentlich subversiv gedachten Kunstformen. Darüber hinaus stellt aktuelle globale Entwicklung der Gentrifizierung neben der postkolonialen Kondition des lusophonen Raums einen logischen und wichtigen Schwerpunkt des Bandes dar. Die reichhaltigen Lektüren von Texten, Fotografien, Filmen, Comics, Postern, Graffiti und Kontexten helfen, über zwei kleine Schwächen des Bandes hinwegzusehen. Erstens wäre es wünschenswert, sozialwissenschaftliche Erkenntnisse über Urbanität - immerhin verstehen sich die urban cultural studies als interdisziplinär - stärker in die Analysen einzubinden, wie es beispielsweise bei Prata oder Scholz der Fall ist. Zweitens könnten ein oder zwei Beiträge über urbane Dynamiken früherer Jahrhunderte das Panorama noch erweitern. Lediglich Martínez-Tejero und Medeiros beziehen zum Beispiel das pombalinische Lissabon in ihre Analysen mit ein. Nichtdestotrotz liegt mit Cities of the Lusophone World ein tadellos redigierter, dank Index sehr leserfreundlicher und, da konsequent auf Englisch verfasst, international orientierter Sammelband vor, der wichtige und drängende Fragestellungen behandelt und neue Themen aufwirft. Dies geschieht bisweilen in Form einer sich politisch klar positionierenden Literatur- und Kulturwissenschaft, was möglicherweise bei einigen Forschenden Unmut auslöst, jedoch die erfreuliche Konsequenz hat, dass viele nicht-kanonische Text- und Kunstformen Beachtung finden, die es allemal verdient haben. 